\title{
Time Series Based Short Term T1DM Prediction of Librepro CGM Sensor Data: A Novel Ensemble Method
}

\author{
Rekha Phadke, Varsha Prasad, H C Nagaraj
}

\begin{abstract}
As per statistics over 30 million in India have been diagnosed with diabetes. There is an enormous need and development to be made to recognize the possible fluctuation of blood glucose before hand with minimal errors and thereby enabling proactive decision making.. The present work details out the algorithms used for glucose prediction and makes a relative assessment of glucose prediction of Librepro Continuous Glucose Monitoring (CGM) sensor data of Type 1 Diabetes Mellitus (T1DM) subjects. For the development and evaluation of the model, 10 days observation data of 10 different subjects with T1DM recorded at every 15 minutes time interval is considered. The model's predictive performance is evaluated for one step ahead (15 minutes prediction horizon), two step ahead (30 minutes prediction horizon) and three step ahead (45 minutes prediction horizon) under univariate glucose prediction model. A novel hybrid data driven model which combines both linear regression and auto regression method is designed and developed for glucose prediction. This novel data driven model gave satisfactory performance metrics of MAPE value of 3.22 and RMSE of 7.38 mg/dl over the complex ARIMA model which requires proper selection of parameters to be chosen beforehand. In this paper an attempt has been made by the author to propose an ensemble method towards data driven model for glucose prediction under time series forecasting.
\end{abstract}

Keywords ARIMA, CGM Sensor, Linear Regression, Overlapping forward window rolling technique, Time Series Forecasting

\section{INTRODUCTION}

Diabetes mellitus is reaching reasonably pandemic degree of magnitude in India. The potential complexity due to diabetes are enormous with respect to its morbidility and death giving rise to noticeable burden in healthcare as well as the family and the society. Also diabetes is occurring among younger generation within our country which is due to transit of people from rural to urban areas. The migration is leading to boom in economy and change in life style which impacts on one's health.

Revised Manuscript Received on August 30, 2019.

* Correspondence Author

Rekha Phadke*, ECE Department, Nitte Meenakshi Institute of Technology, Bangalore, India. Email: rekhaphadke@gmail.com

H C Nagaraj, ECE Department, Nitte Meenakshi Institute of Technology, Bangalore, India. Email: principal@nmit.ac.in

Varsha Prasad, ECE Department, Nitte Meenakshi Institute of Technology, Bangalore, India. Email: varsha.1283@gmail.com

(C) The Authors. Published by Blue Eyes Intelligence Engineering and Sciences Publication (BEIESP). This is an open access article under the CC BY-NC-ND license (http://creativecommons.org/licenses/by-nc-nd/4.0/)
Also there is an unbalance between increase in diabetes and the studies investigating the reason for the condition of this particular disease. Also India is a very large and diverse country with respect to its geographical, socio-economic and ethnicity. Hence this epidemic disease called diabetes is spreading across all the classes of society within the country. This calls for an immediate research and

intervention both at regional and national levels-which should try to break down this potentially picking up problem called as diabetes and also in the upcoming years this catastrophic increase in disease should be fought with various tools ranging from cellular biology to pathophysiology to pharmacology to chemistry, physics, and engineering to transplantation to patient management to health care as per the Fig 1. depicted below.

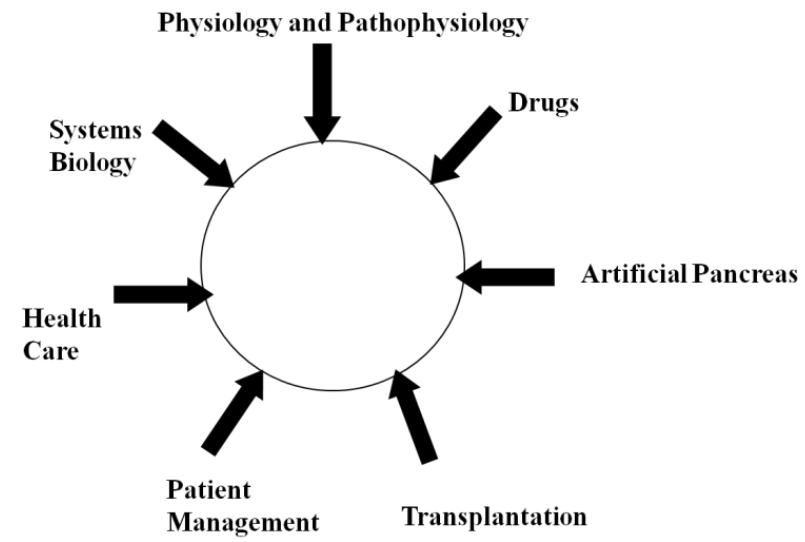

Fig 1. Diabetes control disciplines

Under the patient management, a diabetic patient is provided with a convenient portable device which will help him in assisting in personal care management plan or treatment algorithms that operate upon real time physical measurements and data to generate appropriate dosage amounts for the current administration of medication by injection, constant infusion (insulin pump), or other routes. Diabetes management is a complicated task for patients, who must monitor and control their blood glucose levels using CGM sensors which is mounted on their body in order to avoid serious diabetic complications [2]. It is a difficult task for physicians, who must manually interpret large volumes of blood glucose data to modify therapy to the needs of each patient. 
There are enormous research been carried out which describes emerging applications that employ Artificial Intelligence(AI) to ease this task like case-based decision support for diabetes management; machine learning classification of blood glucose plots; and support vector regression for blood glucose prediction which provides decision support by detecting blood glucose control problems and suggests therapeutic adjustments to correct them or to build a hypoglycemia predictor that could alert patients to dangerously low blood glucose levels in time to take preventive action.. These emerging applications could potentially benefit 20 million patients who are at risk for alarming complications, thereby improving quality of life and reducing health care cost expenditures[3].

There has been a recent outburst of interest in blood glucose (BG) prediction due to its role in closed loop control for the Artificial Pancreas Project (Juvenile Diabetes Research Foundation 2014). In short, an artificial pancreas consists of three components: an insulin pump; a continuous BG monitoring system; and a closed loop control algorithm to tie them together, so that insulin flow can be continuously adjusted to meet patient needs [4-6]. Although still the work is in progress, Time Magazine named the artificial pancreas one of the 25 best inventions of 2013 (Time Magazine 2013). Several recent publications detail related work in BG prediction [7-9]. This motivates many researchers to use AI and machine learning which plays a very important role of forming a bridge between a diabetic patient and the patient management system coupled with physiological modeling. Giovanni sparacino et.al demonstrates glucose predictions by using simple prediction algorithms [10].

Zhao has demonstrated a improved modeling method over a conventional modeling specific to a subject taking into consideration exogenous input which resulted in improved economical modeling method [11]. C Zecchin et al., demonstrated jump neural network for short-time prediction of blood glucose with meal information as input which obtained accurate results [12]. Eleni I. Georga et al., demonstrates glucose prediction combining feature ranking with regression models [13] as well as multivariate prediction based on support vector regression [14]. Kamuran turksoy proposed hypoglycemia early alarm systems based on multivariable models.[15]. Hence glucose prediction models can be classified into three types: physiological, data driven and hybrid [16]. Physiological model requires a good understanding of insulin and glucose metabolism and are commonly used in compartment modeling via simulators. [17]. Data driven models are based on machine learning techniques and a combination of both physiological and data driven model forms the hybrid model. [18-19]

Hence the motivation for this paper is to propose simple time series algorithms for glucose prediction with no exogenous input and hence to evaluate the performance metrics based on only time stamped glucose readings.

\section{DATASET AND DATA PARTITION FOR TIME SERIES MODEL}

Dataset comprising of 15 days of time stamped glucose readings recorded at every 15 minutes interval of 10 subjects were collected from Jnana Sanjeevini diabetes center, Bangalore. A plot of 15 days dataset of a subject is as

depicted in Fig 2. From Fig 2 it is evident that the plot is parallel to X-axis indicating that the dataset is stationary. While the autocorrelation function (ACF) plot of the dataset from Fig 3 indicates that the dataset is non stationary because there is a gradual decrease in the lag which eventually zeroes down. Hence a P-value statistical evaluation is done in $\mathrm{R}$ using kpss test, augmented dickey fuller test and nsdiff () . This confirms whether a differencing is required to transform non stationary data into stationary. Based on the combination of these tests a differencing of one is decided so as to acquire the time series data is proper format.

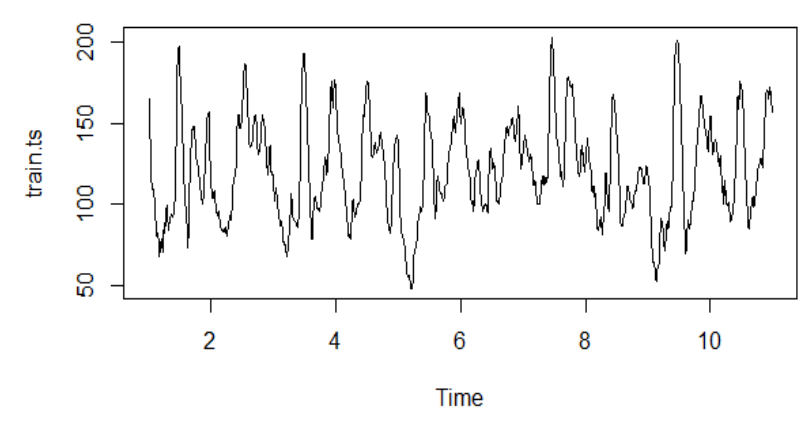

Fig 2: Plot of 15 days T1DM time series data of a subject

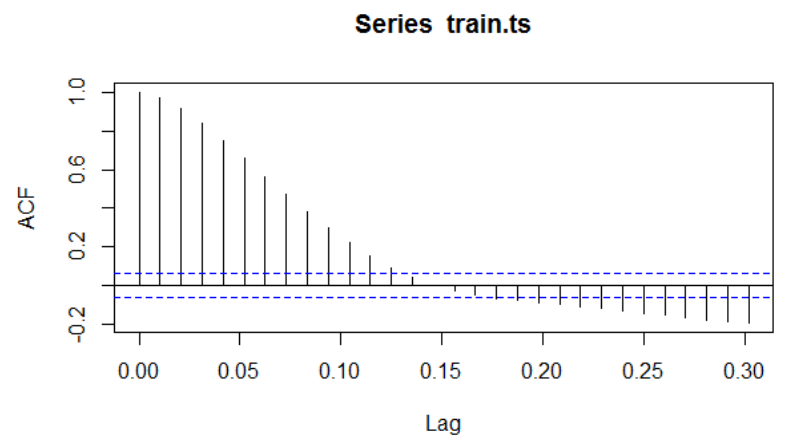

Fig 3: ACF plot of 15 days T1DM time series data of a subject

Dataset is partitioned as per the cross validation technique relevant for time series modeling. Cross validation is a model validation technique for assessing the results of statistical evaluation within time series modeling. It involves partitioning the dataset into subsets, performing the evaluation on one subset (called the training set) and validating the evaluation on the other subset (called the test set). The goal of cross validation is to test the model's ability to predict new data that was not used in estimating it, in order to understand how the model will behave on unseen data. Multiple rounds of cross validation are performed using different partitions and the validation results are averaged over the rounds to give an estimate of model prediction performance. In performing cross validation on time series data , traditional k-fold cross validation will not work since the time series dataset has temporal dependencies and hence test dataset should chronologically appear after train dataset and hence the test data set cannot be arbitrarily chosen since it may lead to data leakage.[20-23].

Published By: Blue Eyes Intelligence Engineering \& Sciences Publication 
Hence we propose a technique called as overlapping forward window rolling technique where in the train and test splits move forward as shown in Fig 4.

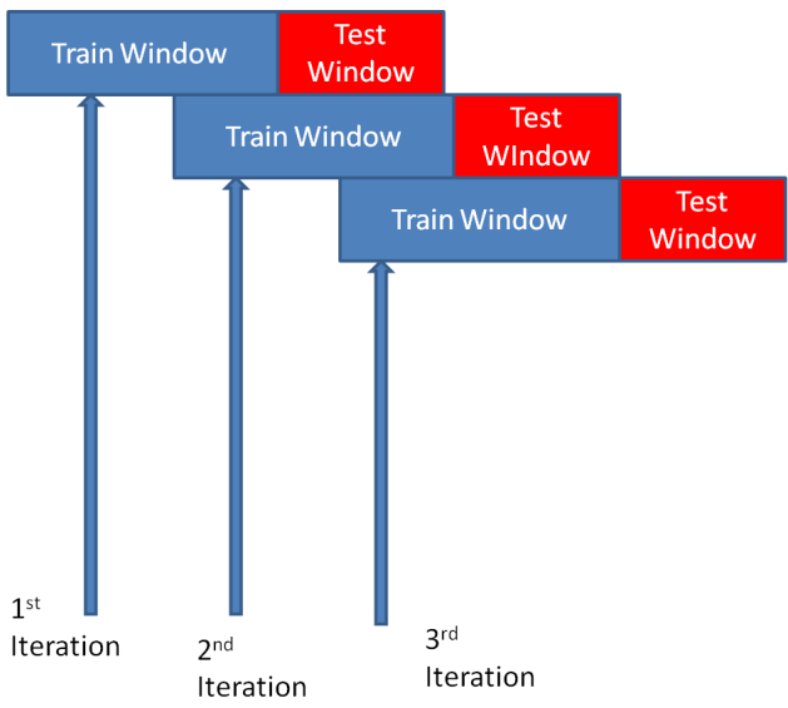

Fig 4: Overlapping Forward Window Rolling technique Here we consider a window size of 12 hours (48 data points), where in :

47 data points: 1 data point: is Train window: Test window for one step ahead prediction, (15 minutes prediction horizon).

46 data points: 2 data points: is Train window :Test window for two step ahead prediction, (30 minutes prediction horizon).

45 data points: 3 data points: is Train window :Test Window for three step ahead prediction,(45minutes prediction horizon).

Hence for total 960 data points (10 days) for each patient, 937 subsets of train and test splits are evaluated (937 iterations) and assessed respectively and are averaged.

\section{TIME SERIES FORECASTING PROCESS}

The method of forecasting begins with objective description. Data is collected and cleaned, and explored using visualization tools. A set of potential forecasting methods are selected, based on the nature of the data. The different methods are applied and compared in terms of forecast accuracy and other measures related to the purpose. The best method is then chosen to produce forecasts [24]. The forecasting process is as shown in Fig 5.

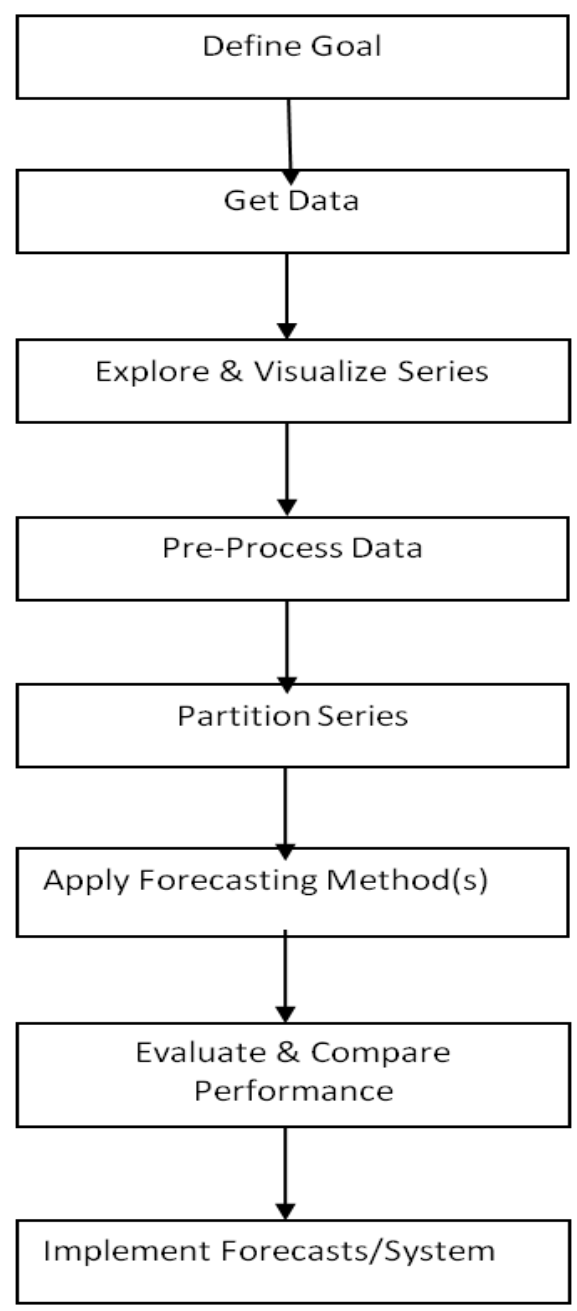

Fig 5: Steps in Forecast Process [19]

\section{The Performance Metrics}

The different performance metrics considered to measure forecast accuracy are

Error

Root Mean Square Error (RMSE)

Mean Absolute Error (MAE)

Mean Absolute Percentage Error (MAPE)

R squared (R2)

Autocorrelation Function (ACF)

Partial Autocorrelation Function (PACF)[25]. A precise explanation of each of the performance metrics is done here.

A. Error:

An error in forecast is the difference between a valid or actual reading and its forecasted value. The error is expressed as:

$\mathbf{e}(\mathbf{t})=\mathbf{y}(\mathbf{t})-\hat{\mathbf{y}}(\mathbf{t})$

Where, e(t) represents forecast error at time instant ' $t$ ', $y(t)$ represents actual value at time instant ' $t$ ' and $\hat{\mathbf{y}}(\mathbf{t})$ represents predicted value at time instant ' $t$ '.

\section{B. Root Mean Square Error:}

RMSE is a scale dependent measure. It is expressed as:

$\operatorname{RMSE}=\sqrt{\operatorname{mean}(\mathbf{e}(\mathrm{t}))^{2}}$ 
RMSE is widely used performance metric as it heavily penalizes bad forecasting.

\section{Mean Absolute Error:}

MAE is also a scale dependent measure. It is expressed as:

MAE $=\operatorname{mean}(|\mathbf{e}(\mathbf{t})|)$

Normally RMSE tend to mask good performance due to a few bad forecasting values. This is avoided in MAE.

\section{Mean Absolute Percentage Error:}

MAPE is a percentage error measure. The percentage error is expressed as:

$\mathbf{p}(\mathbf{t})=100 * \frac{\mathbf{e}(\mathbf{t})}{\mathbf{y}(\mathbf{t})}$

MAPE is given as:

MAPE $=$ mean $(|\mathbf{p}(\mathbf{t})|)$

E. R squared:

$\mathrm{R}$ squared is the squared correlation between the forecast variable and its estimated value. It is expressed as

$\mathbf{R} 2=\sum \frac{(\hat{\mathbf{y}}-\overline{\mathbf{y}}) 2}{(\mathbf{y}-\overline{\mathbf{y}}) \mathbf{2}}$

F. ACF and PACF of a forecast error:

This is very useful in influencing whether there is any remaining pattern in the error (residuals) after a forecasting model has been applied. This is not a measure of accuracy for each, but rather can be used to point out whether a forecasting method could be enhanced.

\section{ERROR GRID EVALUATION}

While the statistical evaluation or the analytical evaluation measures are explained in the above section, these evaluation are not suitable to explain the clinical accuracy. The analytical evaluation is a quantitative measure while clinical accuracy is a qualitative measure. The widely established method for clinical accuracy is error grid evaluation. This compares the measurement from both the CGM sensor and the reference method (model) and plots it on the so-called error grid. The evaluation of an error grid is the purpose of finding the percentage of the data points that fall in each zone of clinical outcome.

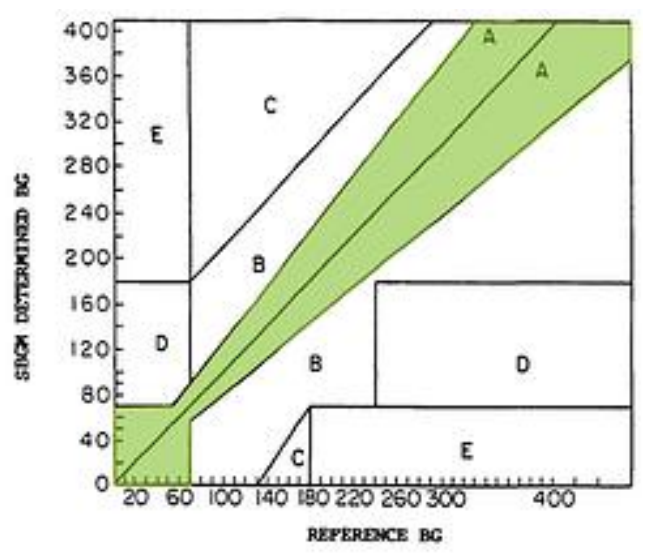

Fig 6: Clark Error-Grid Plot[24]

There are five different zones of the Clark-Error Grid which is as shown in Fig 6 where the different zones are identified as:

- A: Clinically correct decision

- B:Clinically uncritical decision
- C:Overcorrection

- D:Skip a necessary correction

- E:Performing the opposite/wrong "correction" Hence in this paper both analytical and clinical evaluation are carried out.

\section{TIME SERIES FORECASTING MODEL}

A. Linear Regression Model: Linear regression can be setup to capture a time series with a trend and/or seasonality as well as other patterns. The model which is estimated from training period can produce forecasts on future data by inserting the relevant predictor information into the estimated regression equation. For the linear relationship fit between glucose readings and time, we set the output variable y as the forecasted glucose reading and the predictor as the time index $\mathrm{t}$ in the regression model:

$$
\mathrm{y}(\mathrm{t})=\beta_{0}+\beta_{1} \mathrm{t}+\varepsilon
$$

Where $\mathrm{y}(\mathrm{t})$ is the glucose reading at time point $\mathrm{t}$ and $\beta 0, \beta 1$, and $\varepsilon$ are the level, trend and noise component of time series which are modeled using $\mathrm{R}$ using least square estimation (LSE). Seasonality component is not modeled here.

The performance metric obtained by using linear regression for a prediction horizon [PH] of 15minutes, 30minutes and 45 minutes is as listed in Table 1.

Table 1: Performance Metrics of Linear Regression

\begin{tabular}{|c|c|c|c|c|}
\hline PH & RMSE & MAE & MAPE & R2 \\
\hline 15 & 35.52 & 27.84 & 25.68 & 0.56 \\
\hline 30 & 41.34 & 32.39 & 36.72 & 0.483 \\
\hline 45 & 47.2 & 37 & 41.38 & 0.4 \\
\hline
\end{tabular}

As seen from Table 1. The R2 value which is a measure of accuracy for linear regression is not close to 1 and also the MAPE value obtained is not less than 10 percent and RMSE values obtained are not less than $10 \mathrm{mg} / \mathrm{dl}$, all of which indicate that linear regression did not give satisfactory result for the dataset. Also the $\mathrm{P}$ value obtained from $\mathrm{R}$ gave a non significant value.

The plot of forecasted versus actual reading for $\mathrm{PH}$ of 15, 30 and 45 minutes is as shown in Fig 7 . Where rmse values of predicted differ by more than $10 \mathrm{mg} / \mathrm{dl}$ with respect to actual and also has a delay which justifies the values obtained for linear regression which are as shown in Table 1. 


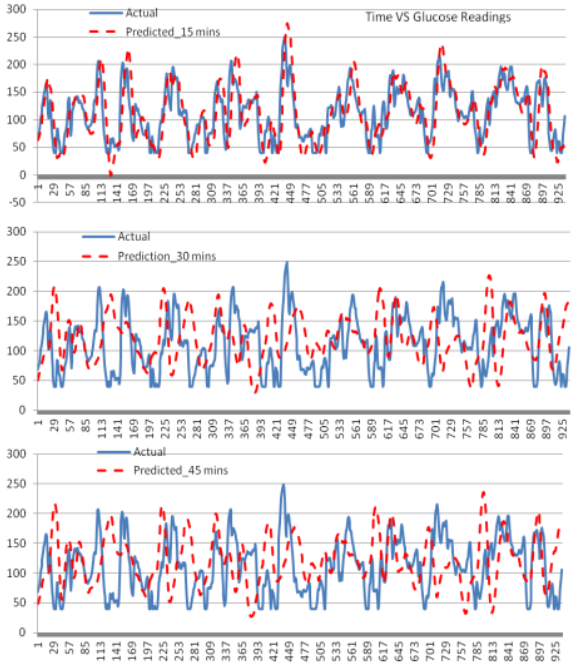

Fig 7. Actual vs Predicted Values for PH of 15, 30 and 45 minutes(Time vs $\mathbf{m g} / \mathbf{d l})$

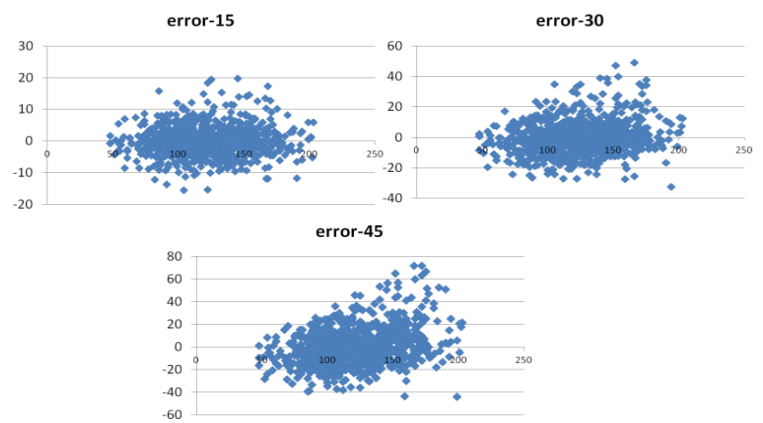

Fig 8: Residual Plots of Predicted value vs Error for PH of 15,30 and 45 minutes

The plot of predicted value vs error residual is shown in Fig 8. It can be seen here that the residual cluster for $\mathrm{PH}$ of 15 ,30 or 45 minutes is not spread and also is unbalanced, indicating that they still carry some information and hence there is a need for improvement. Also the performance metrics of exponential and polynomial regression were checked which gave very much similar results to their linear regression counterpart.

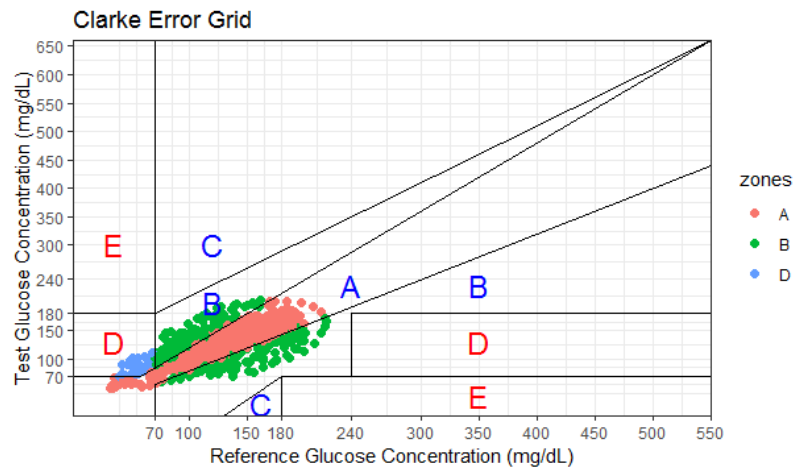

Fig 9: Linear regression applied on Clark-Error Grid

It can be seen from Fig 9 that the data points fall in A, B and $\mathrm{D}$ zones. The data points in $\mathrm{D}$ zone indicate that the necessary corrections to be performed will be skipped and hence this model is of less significance.

\section{B. The ARIMA Model:}

Linear regression used for time series forecasting accounts only for patterns such as trend and seasonality but it will not take into account correlation between neighboring periods. Such correlation called autocorrelation is very useful and help in improving the forecasts. The regression models that directly capture autocorrelation are autoregression models and ARIMA (Autoregressive integrated moving average) models. ARIMA model is hence used to model time series data for forecasting in such a way that

1. A pattern of growth or decline is accounted (auto-regressive part)

2. Rate of change of growth or decline is accounted (Integrated part)

3. Noise between consecutive time points is accounted (Moving Average) (MA)

$\hat{y} \boldsymbol{t}=\boldsymbol{\mu}+\boldsymbol{\varphi 1} \boldsymbol{y t}-1+\cdots+\varphi p y t-p-\theta 1 e t-1 . .-\theta q e t-q(8)$ Where $\boldsymbol{\mu}$ is constant term, is the AR coefficient at lag $\mathrm{k}, \theta_{k}$ is the MA coefficient at lag $\mathrm{k}$, and $e t-k=y t-k-\hat{y} t-k$ is the forecast error that was made at period $\mathrm{t}-\mathrm{k}$.

ARIMA is expressed as ARIMA (p,d,q) where p captures autoregressive part, $\mathrm{d}$ captures integrated part and q captures the moving average part.

Transformations such as log helps in stabilizing the variance and differencing helps to stabilize the mean of a time series by removing changes in the level of a time series and thus eliminating trend or seasonality, since ARIMA works only on stationary signals.

Autocorrelation function (ACF) helps in identifying a time series data as stationary or non stationary and partial autocorrelation function (PACF) gives the partial correlation of a stationary time series with its own lagged values, regressing the values of the time series at shorter lags. Inference of ACF and PACF are as shown in Table 2:

Table 2: ACF and PACF inference

\begin{tabular}{|l|l|l|}
\hline & ACF & PACF \\
\hline AR(p) & $\begin{array}{l}\text { Spikes perish } \\
\text { towards zero }\end{array}$ & $\begin{array}{l}\text { Spikes cutoff to } \\
\text { zero }\end{array}$ \\
\hline MA(q) & $\begin{array}{l}\text { Spikes cutoff to } \\
\text { zero }\end{array}$ & $\begin{array}{l}\text { Spikes perish } \\
\text { towards zero }\end{array}$ \\
\hline ARMA(p,q) & $\begin{array}{l}\text { Spikes perish } \\
\text { towards zero }\end{array}$ & $\begin{array}{l}\text { Spikes perish } \\
\text { towards zero }\end{array}$ \\
\hline
\end{tabular}

Hence using ACF and PACF inference as shown in Table 2 , the model order $\mathrm{p}, \mathrm{q}$ for ARIMA is identified. After this estimation of parameters $\mathrm{C}, \emptyset 1, \varnothing 2, \ldots \emptyset p$ are done using maximum likelihood estimation(MLE) which is similar to least square estimation obtained by minimizing

$\sum_{t-1}^{T} e_{t}^{2}$

The ARIMA () command in R allows MLE estimation.

ARIMA model also uses information criteria like Akaike's information criteria (AIC), Corrected AIC (AICc) and Bayesian information criterion (BIC) to measure statistical model. It basically quantifies the goodness of a fit. Good models are obtained by minimizing either AIC, AICc or BIC. Hence Box-Jerkins Methodology is followed for ARIMA model as shown in Fig 10: 
Table 3: Performance metrics of ARIMA model

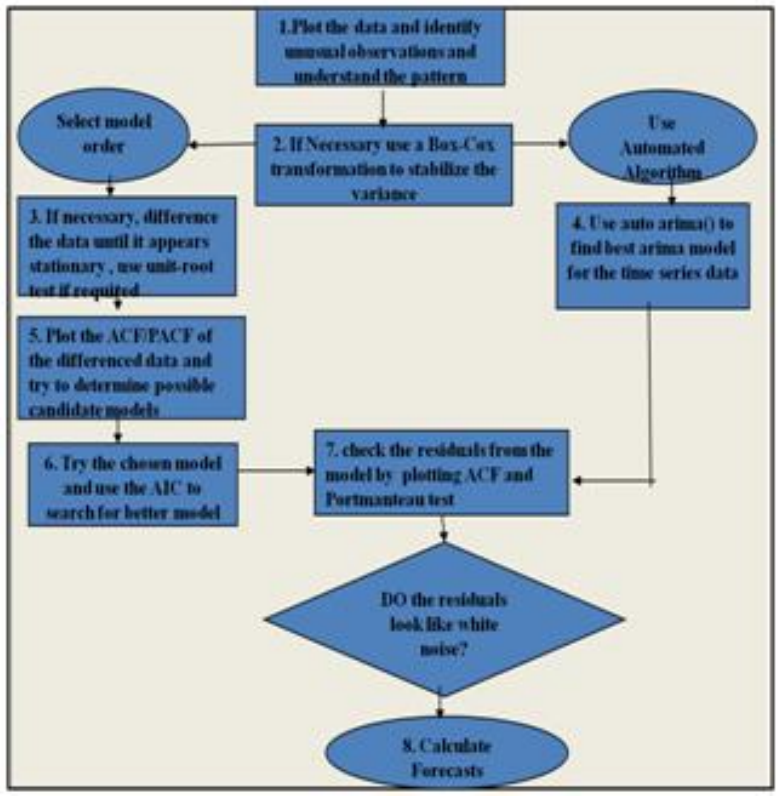

Fig 10: Box-jerkins flow chart for ARIMA modeling [19]

The ACF and PACF plot are as shown in Fig 11 and 12 respectively.

Series dataset10.ts

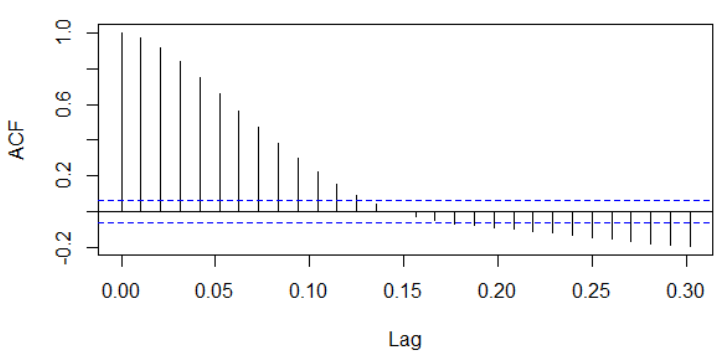

Fig 11: ACF plot of the dataset

Series dataset10.ts

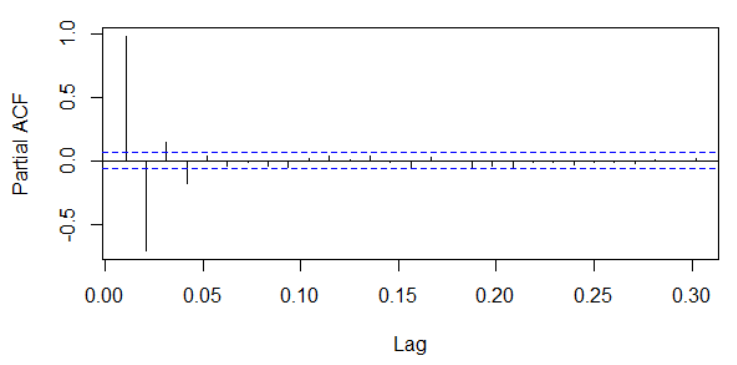

Fig 12: PACF plot of the dataset

Since from Fig 11 and Fig 12 it is seen that ACF spikes perish towards zero and PACF spikes cutoff to zero, it infers that ARIMA model requires only $\mathrm{p}$ and $\mathrm{d}$ for its modeling which is as shown in Table 2. Hence an ARIMA model with ARIMA (p, d,0) is chosen for the dataset.

The performance metrics obtained by ARIMA model ARIMA(2,1,0) for $\mathrm{PH}$ of 15 minutes, 30minutes and 45 minutes are as listed in Table 3.

\begin{tabular}{|c|c|c|c|}
\hline $\begin{array}{c}\text { PH } \\
(\mathrm{min})\end{array}$ & RMSE & MAE & MAPE \\
\hline 15 & 7.07 & 5.12 & 3.98 \\
\hline 30 & 15.89 & 11.58 & 9.37 \\
\hline 45 & 23.29 & 17.47 & 13.87 \\
\hline
\end{tabular}

As seen from Table 3, the MAPE value using ARIMA model is quite satisfactory since it is less than 10 percent error for $\mathrm{PH}$ of 15 and 30 minutes. Also the RMSE value for PH of 15 minutes is less than $10 \mathrm{mg} / \mathrm{dl}$. Hence ARIMA model proved to be better than linear regression for the dataset.

The plot of forecasted versus actual reading for $\mathrm{PH}$ of 15 , 30 and 45 minutes is as shown in Fig. 13. which indicates that the predicted value for $\mathrm{PH}$ of 15 minutes is close to actual value and $\mathrm{PH}$ of 30 and 45 minutes show satisfactory results with sudden spikes in predicted values.
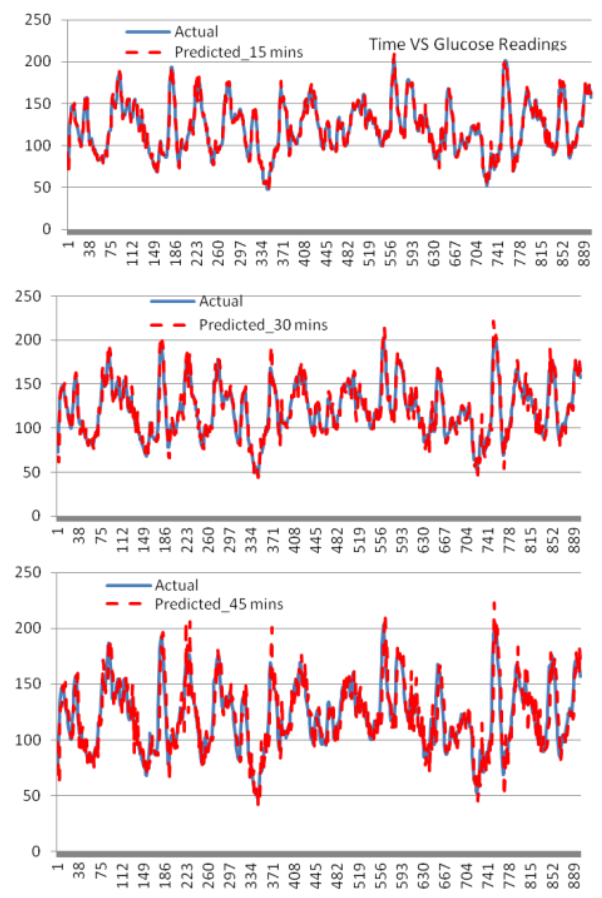

Fig 13: Actual vs Predicted Values for PH of 15, 30 and 45 minutes (Time vs mg/dl)

A residual plot for $\mathrm{PH}$ value of 15, 30 and 45 minutes is also plotted which is as shown in Fig 14. 


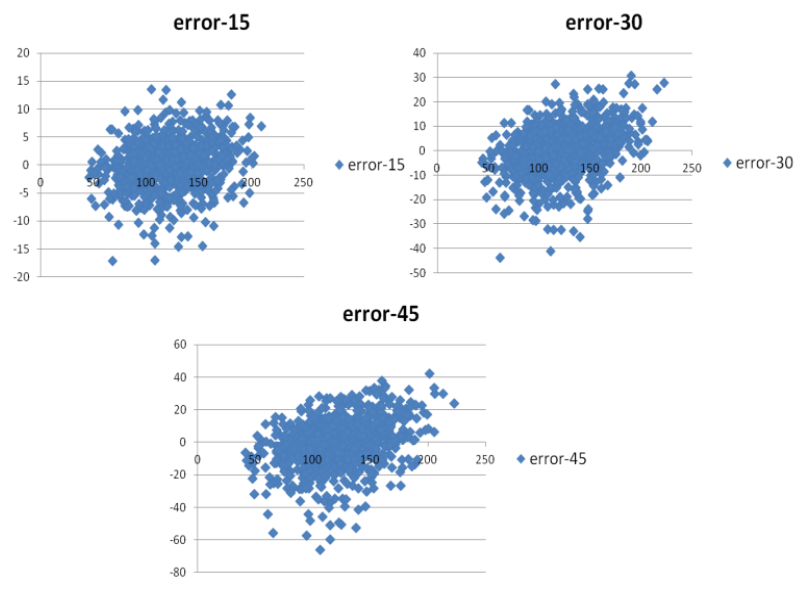

Fig 14: Plot of Predicted vs Error for PH of 15, 30 and 45 minutes

From Fig 14 we can infer that residual cluster for $\mathrm{PH}$ of 15 minutes is balanced while the residual cluster of $\mathrm{PH}$ for 30 minutes and $\mathrm{PH}$ for 45 minutes is spread but unbalanced across $\mathrm{x}$ and $\mathrm{y}$ axis proving that ARIMA model obtained good result for $\mathrm{PH}$ of 15 minutes while satisfactory result for $\mathrm{PH}$ of 30 and 45 minutes.

Also the ACF plot of residual is obtained which has a mean value close to zero indicating that the residual obtained is white noise as shown in Fig 15.

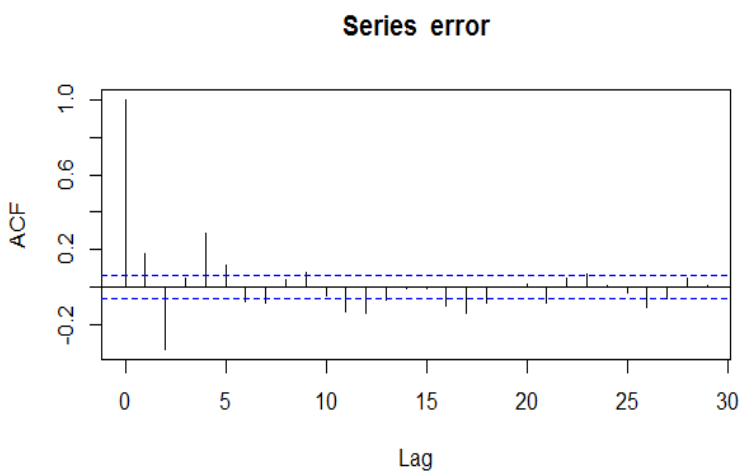

Fig 15: ACF plat of residual of the dataset

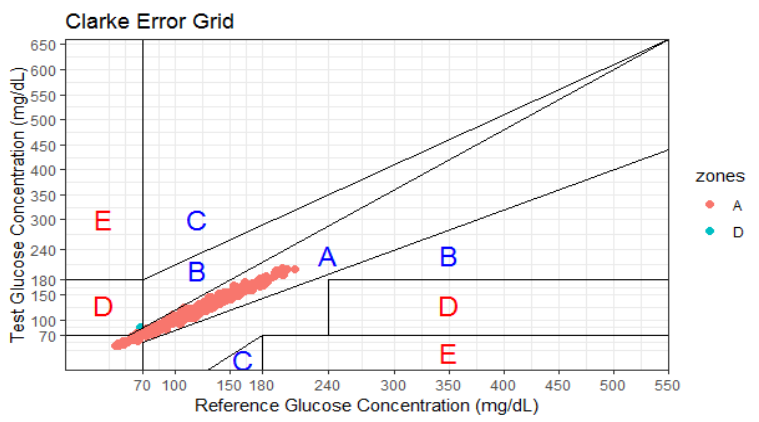

Fig 16: ARIMA applied on Clark-Error Grid

It can be seen from Fig 16 that the data points fall in A and D zones. The data points in $\mathrm{D}$ zone indicate that the necessary corrections to be performed will be skipped. But the data points in D zone are hardly any indicating that ARIMA is better than linear regression.

\section{.C. A Novel Ensemble Model:}

ARIMA model creates larger flexible forecasting models but also requires much more complicated statistical expertise. Hence the author goes for an ensemble method which reduces the statistical complexity.
This proposed method is captured by constructing a second level of forecasting model for residuals.The steps are as follows:

1. Generate k-step ahead forecast of the series $\mathrm{y}_{\mathrm{t}+\mathrm{k}}$, using a forecasting method (author has used linear regression).

2. Generate k-step ahead forecast of the forecast error $\left(e_{t+k}\right)$, using an AR model.

3. Improve the initial k-step ahead forecast of the series by adjusting it according to its forecasted error:

Improved $\mathrm{y}_{\mathrm{t}+\mathrm{k}}{ }^{*}=\mathrm{y}_{\mathrm{t}+\mathrm{k}}+e_{t+k}$

This three step process results in fitting low-order AR model to the series of residuals which is then used to forecast future residuals. To fit an AR model to the series of residuals, we first examine the autocorrelations of the residual series. We then choose the order of the AR model according to the lags in which the autocorrelation appears. Often when autocorrelation appears at lag- 1 and higher, it is sufficient to fit an AR (1) model of the form

$e_{t}=\beta_{0}+\beta_{1} e_{t-1}+\varepsilon \quad$ (11)

Where $e_{t}$ denotes the residual forecast error at time t.

Series $\mathrm{x}$

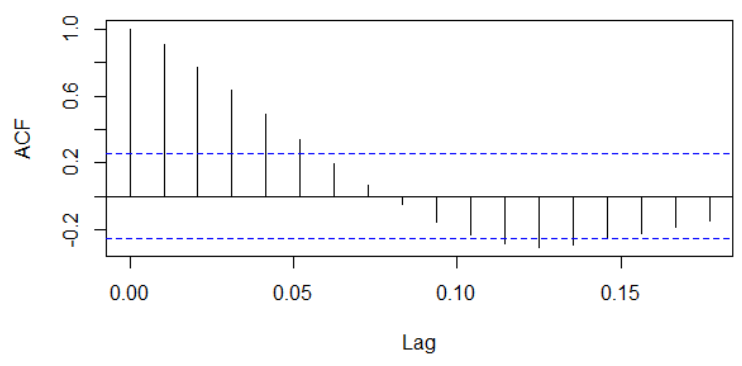

Fig 17: ACF output of autocorrelations of residual series after fitting regression

From Fig 17 it could be seen that although the autocorrelation appears to be large at every lag, it is likely that AR (1) could arrest all these relationships. The AR (1) model is then fitted to the residual series.

The plot of forecasted versus actual reading for $\mathrm{PH}$ of 15, 30 and 45 minutes is as shown in Fig. 14.

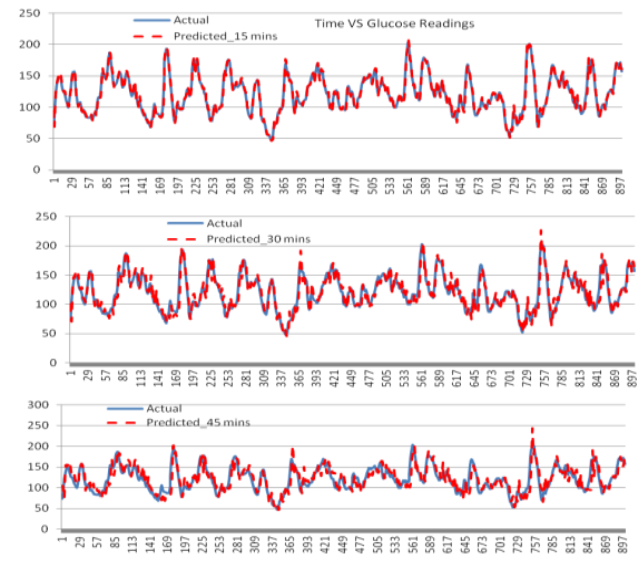

Fig 18: Plot of forecast vs actual reading for $\mathrm{PH}$ of 15,30 and 45 minutes

From Fig 18 we can infer that the results obtained through ensemble method is very closer to the ARIMA model and also it clearly indicates that the forecasted value is almost close to actual value for $\mathrm{PH}$ of 15 minutes.

Published By: 
Table 4: Performance metrics of Novel Method

\begin{tabular}{|c|c|c|c|}
\hline $\begin{array}{c}\text { PH } \\
(\mathrm{min})\end{array}$ & RMSE & MAE & MAPE \\
\hline 15 & 7.38 & 5.47 & 3.22 \\
\hline 30 & 17.09 & 12.9 & 10.27 \\
\hline 45 & 26.3 & 20.09 & 16.06 \\
\hline
\end{tabular}

As seen from Table 4, the MAPE value using the novel model is quite satisfactory since the values obtained are less than and approximately 10 percent error for $\mathrm{PH}$ of 15 and 30 minutes. Also the RMSE value for $\mathrm{PH}$ of 15 minutes is less than $10 \mathrm{mg} / \mathrm{dl}$. Hence the Novel Ensemble Model proved to be very close to ARIMA model which can be further improved by including external information.

Finally to confirm whether we have indeed achieved for autocorrelation in the series and no more information remains in the series we examine the autocorrelation of residuals-of-residuals as shown in Fig 19.

Series fit.pred2\$residuals

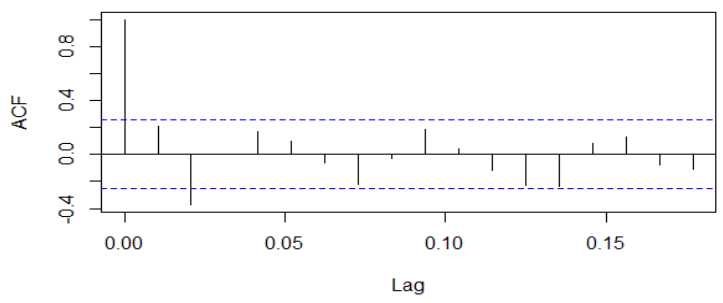

Fig19:Autocorrelation of residuals-of-residuals series

From Fig 19 it is clear that the addition of AR(1) model has effectively captured the autocorrelation information, since ACF values are within the threshold.

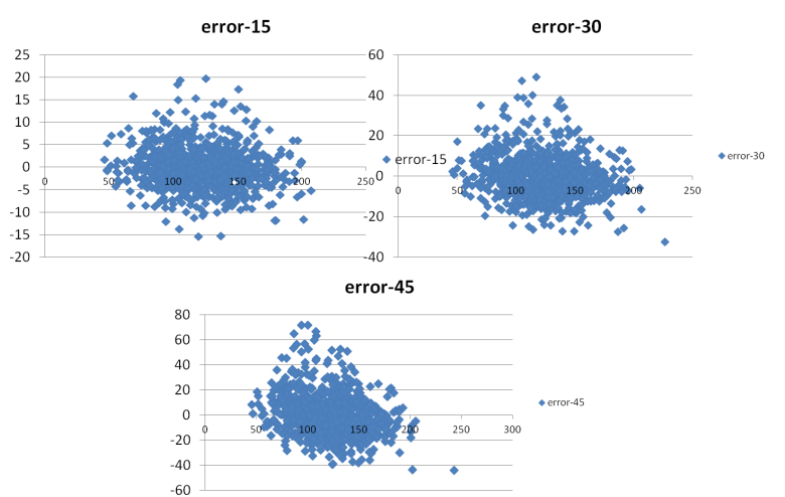

Fig 20: Residual plot of Novel Ensemble Model for PH of 15, 30 and 45 minutes

Also Fig 20 shows the residual plot for $\mathrm{PH}$ of 15,30 and 45 minutes respectively, with the first plot showing balanced residual cluster while the other two plots showing unbalanced residual cluster with similarities as in ARIMA model. Hence this Novel Ensemble Model behaves very similar to ARIMA model though it is a very simple model technique when compared to the sophisticated ARIMA.

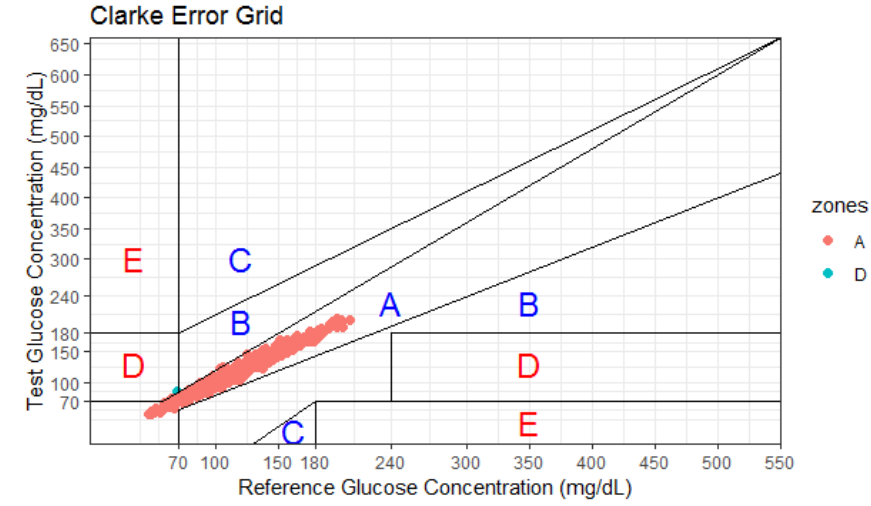

\section{Fig 21: Novel Ensemble Model applied on Clark-Error Grid}

It can be seen from Fig 21 that the data points fall in A and D zones. The data points in $\mathrm{D}$ zone indicate that the necessary corrections to be performed will be skipped. But the data points in D zone are hardly any and also match the Clark-Error Grid of ARIMA indicating that this Novel Ensemble Model coincides with ARIMA in its performance.

\section{RESULT AND DISCUSSION}

Table 5 gives the evaluation of all the algorithms implemented for a $\mathrm{PH}$ of 15 minutes.

Table 5. Performance Evaluation Metrics Juxtaposition Table of ML Algorithms implemented on LIBREPRO Dataset

\begin{tabular}{|c|c|c|c|}
\hline Algorithm & RMSE & MAE & MAPE \\
\hline $\begin{array}{c}\text { Linear } \\
\text { Regression }\end{array}$ & 35.52 & 27.84 & 25.68 \\
\hline ARIMA & 7.07 & 5.12 & 3.98 \\
\hline $\begin{array}{c}\text { Novel } \\
\text { Ensemble } \\
\text { Model }\end{array}$ & 7.38 & 5.47 & 3.22 \\
\hline
\end{tabular}

It is seen from Table 5 that since Linear regression captures only trend and seasonality but not the correlation between neighboring points the values obtained are not satisfactory and hence such a model cannot be used for Glucose prediction.

While ARIMA is a type of regression model which can be used to quantify the correlation between neighboring values in a time series, called as autocorrelation. Hence this type of model is very useful in improving forecast accuracy by making use of the information contained in the autocorrelation which is beyond trend and seasonality. The values obtained for ARIMA model in Table 5 clearly indicates that it is a better model than linear regression.

But while ARIMA is better for forecasting, it also requires much more statistical proficiency to use it as a forecasting model. Hence our Novel Ensemble Model overcomes these sophistications in a straightforward manner to be relevant in the context of forecasting, 
which provides significant improvement in short-term forecasting. Table 5 results for Novel Ensemble Model has obtained values very close to the complex ARIMA model.

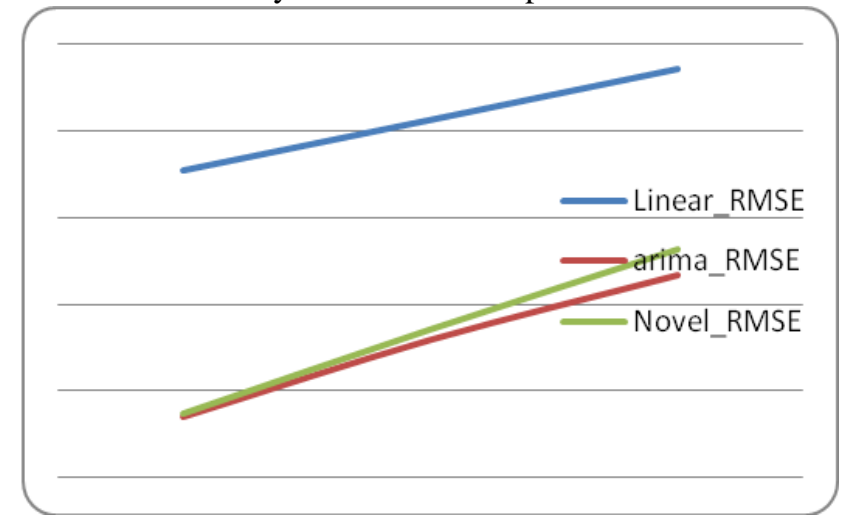

Fig 22: RMSE plot for all the algorithms at different $P H$ intervals

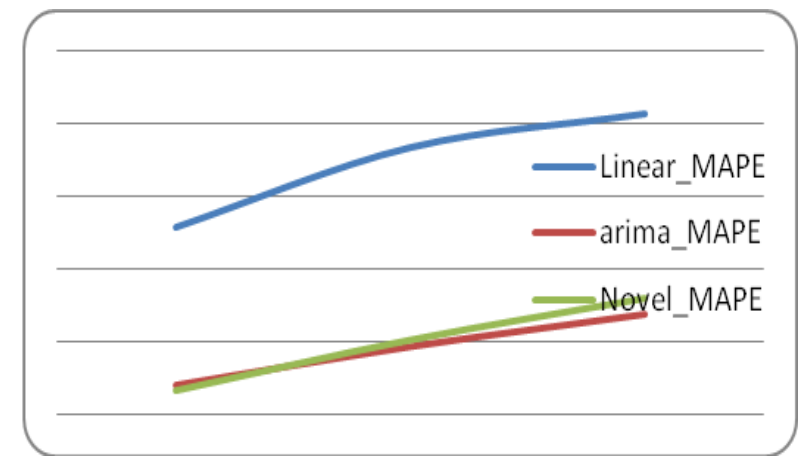

Fig 23: MAPE plot for all the algorithms at different $\mathrm{PH}$ intervals Fig 22 and 23 depicts the RMSE and MAPE plot for all the algorithms implemented for a PH of 15 minutes. The RMSE and MAPE of ARIMA and Novel Ensemble Model almost coincide which asserts that this Novel Ensemble Model can be implemented for Librepro CGM dataset for glucose prediction since it is easier to implement it when compared to ARIMA model.

\section{CONCLUSION}

The Novel Ensemble method outlined in this paper provides a way to improve the accuracy of prediction, as well as to stiffen the probability bands on them by understanding the driving dependencies, building the regression(s), and forecasting the dependent variables, followed by forecasting of the residuals. Because the residuals get forecasted unlike Linear regression or Arima, this technique is more robust and more precise than any methods used currently in forecasting dependent variables.

\section{ACKNOWLEDGMENT}

We sincerely thank Vibha Rao, from Jnana Sanjeevini, Bangalore for proving us with Librepro Dataset and also Dr. Abhijit Bhogaraj, Endocrinologist who authenticated our work and results.

\section{REFERENCES}

1. Balakrishnan NP, Rangaiah GP, Samavedham L. "Personalized blood glucose models for exercise, meal and insulin interventions in type 1 diabetic." Annual International Conference of the IEEE Engineering in Medicine and Biology Society. 2012. 1250-1253.
2. Bunescu, Razvan \& Struble, Nigel \& Marling, Cindy \& Shubrook, Jay \& Schwartz, Frank. "Blood Glucose Level Prediction Using Physiological Models and Support Vector." Proceedings of the 2013 12th International Conference on Machine Learning and Applications - Volume 01. 2013. 135-140.

3. C. Bergmeir and J. M. Benítez., May 2012. ISSN 0020-0255. "On the use of cross-validation for time series predictor evaluation. Inf. Sci.,." 2012, 191:192-213.

4. Chunhui Zhao, Chengxia Yu. "Rapid Model Identification for Online Subcutaneous Glucose Concentration Prediction for New Subjects With Type I Diabetes." IEEE Transactions on Biomedical Engineering 62, no. 5 (2015).

5. Cindy Marling, Matthew Wiley, Razvan Bunescu. "Emerging Applications for Intelligent Diabetes Management." AI Magazine, 2012: 67-78.

6. cooper, colleen. Patient management of diabetes treatment. United States of America Patent US20050197553A1. 09 08, 2005.

7. Estrada GC, Kirchsteiger H, Eric R.Am Control Conf. "Innovative Approach for Online Prediction of Blood Glucose Profile in Type 1 Diabetes Patients." Am Control Conf. 2010. 2015-2020.

8. Eyal Dassau, Christian Lowe, Cameron Barr, Eran Atlas, Moshe Phillip. "Closing the loop." International Journal of Clinical Practice Issue- Wiley Online Library 66, no. s175 (feb 2012).

9. Georga EI, Protopappas VC , Polyzos D , Fotiadis DI. "Evaluation of short-term predictors of glucose concentration in type 1 diabetes combining feature ranking with regression models." Medical \& Biological Engineering \& Computing 53, no. 12 (2015): 1305-1318.

10. Georga, Eleni \& Protopappas, Vasilios \& Ardigò, Diego \& Marina, Michela \& Zavaroni, Ivana \& Polyzos, Demosthenes \& Fotiadis, Dimitrios. "Multivariate Prediction of Subcutaneous Glucose Concentration in Type 1 Diabetes Patients Based on Support Vector Regression." IEEE transactions on information technology in biomedicine : a publication of the IEEE Engineering in Medicine and Biology Society., 2012.

11. Giovanni Sparacino, Francesca Zanderigo,Stefano Corazza,Alberto Maran,Andrea Facchinetti,Claudio Cobelli. "Glucose Concentration can be Predicted Ahead in Time From Continuous Glucose Monitoring Sensor Time-Series." IEEE Transactions on Biomedical Engineering 54, no. 5 (2007): 931-937.

12. Iván Contreras, Silvia Oviedo , Martina Vettoretti, Roberto Visentin, Josep Vehí. "Personalized blood glucose prediction: A hybrid approach." PLOS ONE 12, no. 11 (2017).

13. Jensen MH, Christensen TF, Tarnow L, Seto E, Dencker Johansen M, Hejlesen OK. "Real-time hypoglycemia detection from continuous glucose monitoring data of subjects with type 1 diabetes. ." Diabetes Technol Ther. 15, no. 7 (2013): 538-43.

14. Kamuran Turksoy, Elif S Bayrak, Lauretta Quinn, Elizabeth Littlejohn, Derrick Rollins, and Ali Cinar. "Hypoglycemia Early Alarm Systems Based On Multivariable Models." Ind Eng Chem Res. 52, no. 35 (2014).

15. Kevin Plis, Razvan Bunescu,Jay Shubrook,Frank Schwartz. "A Machine Learning Approach to Predicting Blood Glucose Levels for Diabetes Management." Modern Artificial Intelligence for Health Analytics, 2014: 35-39.

16. Kumar, Kanakatte Mylariah Prasanna. "Incidence trends for childhood type 1 diabetes in India." Indian Journal of Endocrinology and Metabolism / 19, no. 5 (2015): 19-34.

17. Man CD, Micheletto F, Lv D, Breton M, Kovatchev B, Cobelli C. "The UVA/PADOVA Type 1 Diabetes Simulator: New Features." $J$ Diabetes Sci Technol. 8, no. 1 (2014): 26-34.

18. Rekha Phadke, Varsha Prasad, H C Nagaraj, Nagesh K P. "Glucose Level Prediction of LIBREPRO CGM Sensor Data Using Machine Learning Algorithm for Enhanced Diabetes Mellitus Management", International Journal of Computer Sciences ...." International Journal of Computer Sciences and Engineering 7, no. 5 (2019): 1571-1582.

19. Shmueli, G. Practical Time Series Forecasting: A Hands-On Guide [2nd Edition]. Axelrod Schnall, 2011.

20. Simon, S. Varma and R. "Bias in error estimation when using cross-validation for model selection. ." BMC Bioinformatics 7, no. 1 (2006).

21. Spyros Makridakis, Steven C Wheelwright, Rob J Hyndman Forecasting methods and applications. John wiley \& sons, 2008.

22. Tashman, L. J. "Out-of-sample tests of forecasting accuracy: an analysis and review." 2000: 437-450. 
23. Wang Q, Molenaar P , Harsh S , Freeman K, Xie J , Gold C , Rovine M , Ulbrecht J . "Personalized State-space Modeling of Glucose Dynamics for Type 1." J Diabetes Sci Technol 8, no. 2 (2014): 331-345.

24. William L Clarke, MD, Daniel Cox, PhD, Linda A Gonder-Frederick $\mathrm{PhD}$, William Carter, PhD and Stephen L Pohl, MD. "Evaluating Clinical Accuracy of Systems for Self-Monitoring of Blood Glucose." Diabetes Care 10, no. 5 (1987): 622-628.

25. Zecchin C, Facchinetti A, Sparacino G, Cobelli C. "Jump neural network for online short-time prediction of blood glucose from continuous monitoring sensors and meal information." Comput Methods Programs Biomed. 113, no. 1 (2014): 144-52.

26. Zecchin C, Facchinetti A, Sparacino G, Cobelli C. "Reduction of number and duration of hypoglycemic events by glucose prediction methods: a proof-of-concept in silico study." Diabetes Technol Ther. 15, no. 1 (2013): 66-77.

\section{AUTHORS PROFILE}

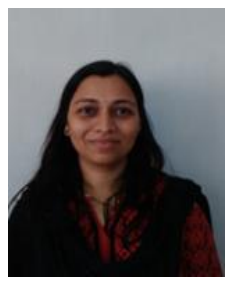

Rekha Phadke is a PhD student under University of Mysore. She works in NMIT, Bangalore. She holds a degree in ECE from VTU and M.Tech from VTU. She has over 15 years of teaching experience. She has published 4 research papers in well reputed journals.

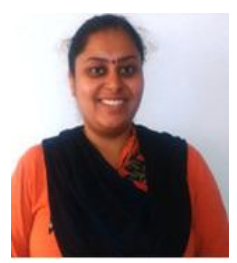

Varsha Prasad is a PhD student under VTU. She works in NMIT, Bangalore. She holds a degree in Instrumentation Technology from VTU and M.Tech from VTU. She has over 13 years of teaching experience. She haspublished 9 research papers in well reputed

SCOPUS indexed journals.

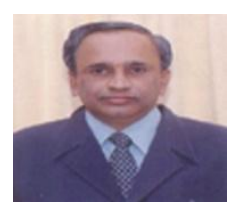

H. C. Nagaraj is currently working as the Principal of NMIT, Bangalore. He holds B.E (Electronics and Communications) degree from the University of Mysore, M.E(Communication systems) degree from P.S.G College of Technology, Coimbatore and Ph. D (Biomedical Signal Processing and Instrumentation) from Indian Institute of Technology, Chennai. He has teaching experience of almost 4 decades, Dr. H. C. Nagaraj aims to bring NMIT among the top 50 education institutes according to NIRF ranking. He has massive experience of 37 years in teaching. He has visited 15 countries and studied the University Engineering Education System, involving various laboratories, centers of excellence etc. He has published 30 papers nationally and internationally. 